\title{
Coincident Generation of Pyramidal Neurons and Protoplasmic Astrocytes in Neocortical Columns
}

\author{
Sanjay Magavi, ${ }^{1}$ Drew Friedmann, ${ }^{2}$ Garrett Banks, ${ }^{3}$ Alberto Stolfi, ${ }^{2}$ and Carlos Lois ${ }^{4}$ \\ ${ }^{1}$ Department of Neurology, Vertex Pharmaceuticals, Cambridge, Massachusetts 02139, ${ }^{2}$ Center for Integrative Genomics, Division of Genetics, Genomics \\ and Development, Department of Molecular and Cell Biology, University of California, Berkeley, Berkeley, California 94720 , ${ }^{3}$ Columbia University, New \\ York, New York 10027, and ${ }^{4}$ Neurobiology Department, University of Massachusetts Medical School, Worcester, Massachusetts 01605
}

Astrocytes, one of the most common cell types in the brain, are essential for processes ranging from neural development through potassium homeostasis to synaptic plasticity. Surprisingly, the developmental origins of astrocytes in the neocortex are still controversial. To investigate the patterns of astrocyte development in the neocortex we examined cortical development in a transgenic mouse in which a random, sparse subset of neural progenitors undergoes CRE/lox recombination, permanently labeling their progeny. We demonstrate that neural progenitors in neocortex generate discrete columnar structures that contain both projection neurons and protoplasmic astrocytes. Ninety-five percent of developmental cortical columns labeled in our system contained both astrocytes and neurons. The astrocyte to neuron ratio of labeled cells in a developmental column was 1:7.4, similar to the overall ratio of 1:8.4 across the entire gray matter of the neocortex, indicating that column-associated astrocytes account for the majority of protoplasmic astrocytes in neocortex. Most of the labeled columns contained multiple clusters of several astrocytes. Dividing cells were found at the base of neuronal columns at the beginning of gliogenesis, and later within the cortical layers, suggesting a mechanism by which astrocytes could be distributed within a column. These data indicate that radial glia are the source of both neurons and astrocytes in the neocortex, and that these two cell types are generated in a spatially restricted manner during cortical development.

\section{Introduction}

Historically, astrocytes have been considered simple support cells with little functional specificity or regional identity. The current models regarding the generation of cortical astrocytes reflect this assumed uniformity. Several works suggest that the majority of cortical astrocytes are generated the subventricular zone (SVZ) (Levison et al., 1993; Levison and Goldman, 1993; Luskin and McDermott, 1994; Marshall and Goldman, 2002), from where they migrate and populate cortex (Zerlin et al., 1995; Kakita and Goldman, 1999; Kakita et al., 2003). This model indicates that the majority of astrocytes arise from precursors that disperse widely, without spatial specificity.

In addition, radial glia are also thought to contribute a smaller fraction of the astrocyte population within the cortex. It has been reported that radial glia, after generating projecting neurons, retract their processes and differentiate into protoplasmic astrocytes (Schmechel and Rakic, 1979; Voigt, 1989; Culican et al., 1990; Gressens et al., 1992). Since these data suggested that individual radial glia transform into individual astrocytes, it was assumed that their contribution to the overall population of

Received July 11, 2011; revised Jan. 30, 2012; accepted Feb. 9, 2012.

Author contributions: S.M., A.S., and C.L. designed research; S.M., D.F., G.B., A.S., and C.L. performed research; S.M., G.B., A.S., and C.L. contributed unpublished reagents/analytic tools; S.M., D.F., G.B., A.S., and C.L. analyzed data; S.M. and C.L. wrote the paper.

This work was supported by the David and Lucile Packard Foundation to C.L.

Correspondence should be addressed to Carlos Lois, Neurobiology Department, University of Massachusetts Medical School, 364 Plantation Street, LRB 770Y, Worcester, MA 01605. E-mail: Carlos.Lois@umassmed.edu.

DOI:10.1523/JNEUROSCI.3560-11.2012

Copyright $\odot 2012$ the authors $\quad 0270-6474 / 12 / 324762-11 \$ 15.00 / 0$ cortical astrocytes was very small. However, other experiments examining neural development were not consistent with these findings. For instance, It has been reported that radial-glia derived columns in the cortex are composed exclusively of pyramidal neurons, without astrocytes (Luskin et al., 1988; Price and Thurlow, 1988; Parnavelas et al., 1991; Mione et al., 1994; Tan et al., 1998; McCarthy et al., 2001; Reid and Walsh, 2002; Wilkie et al., 2004).

To investigate the development and distribution of astrocytes in the brain we performed fate mapping in the brains of transgenic mice that expresses Cre sparsely and produce labeling of a small and apparently random set of brain precursors and their progeny. We observe that cortical progenitors give rise to discrete columnar structures that contain both projection neurons and protoplasmic astrocytes. Most columns of neurons contained multiple clusters of astrocytes, and $98 \%$ of labeled astrocytes were found in or within $50 \mu \mathrm{m}$ of a labeled neuronal column. The astrocyte to neuron ratio of labeled cells in a developmental column was similar to the overall ratio across the entire neocortex, indicating that column-associated astrocytes account for the majority of protoplasmic astrocytes in the neocortex. These data suggest that cortical protoplasmic astrocytes are generated in a spatially restricted manner from the precursors that also give rise to developmental columns of pyramidal neurons.

\section{Materials and Methods}

Generation of transgenic mice

We generated several lines of enhancer trap mice carrying an enhancer detector cassette expressing Cre recombinase under the transcriptional control of the minimal promoter (129 base pairs) of the mouse Thy-1.2 
gene. The transgenics were generated via lentiviral vectors, allowing us to rapidly generate large numbers of animals with random integration sites. The transgene integrated into different sites in each of the thyl.2-cre lines, giving rise to different patterns of recombination upon breeding with reporter mice. We crossed these thy1.2-cre mice to the reporters Z/EG or mch/lox/GFP mice (gift from S. Dymecki, Harvard Medical School, Cambridge, MA), which, upon Cre/loxP recombination, exhibit GFP expression.

\section{Immunohistochemistry}

All experiments were performed in accordance with the National Institutes of Health Guide and Use of Laboratory Animals, and approved by the Massachusetts Institute of Technology Committee on Animal Care. For the analysis of the progeny of thy1.2-cre lines we used animals of either sex, and did not observe any obvious difference between the distribution of GFP + cells between males and females. Mice were killed following a lethal dosage of Avertin. Animals were perfused with PBS followed by $3 \%$ paraformaldehyde. Brains were postfixed in paraformaldehyde overnight at $4^{\circ} \mathrm{C}$ and sectioned at $50 \mu \mathrm{m}$ using a Leica VT1000 vibrating microtome. The sections were immunohistochemically stained with antibodies against BrdU (Accurate Chemical OBT0030G, 1:500), GFP (Aves Labs GFP-1020, 1:8000), NeuN (Millipore Bioscience Research Reagents MAB377, 1:400), GFAP (Sigma G9269, 1:100), S100B (Abcam ab868, 1:2000), MBP (Covance SMI94, 1:1000), Nestin (Abcam Ab6142, 1:500) and Zebrin (Santa Cruz Biotechnology sc-12065, 1:100). To expose the BrdU antigen, we washed sections in $2 \mathrm{M} \mathrm{HCl}$ for $2 \mathrm{~h}$. at room temperature. We used species-appropriate Invitrogen highly crossadsorbed Alexa Fluor 488- and Alexa Fluor 546-conjugated secondary antibodies. Antibodies generated in different species were used in multilabeling experiments to minimize cross-reactivity. Primary and secondary antibodies were diluted in a blocking solution containing $0.3 \%$ Triton, $8 \%$ goat serum and $0.3 \%$ BSA diluted in PBS. Figures were assembled using Adobe Photoshop and Illustrator.

\section{Cell counting}

Quantification of cells was performed using an Olympus IX70 microscope, a Q-imaging Retiga 1300i camera, and Neurolucida software with the virtual slice extension module.

Location of astrocytes relative to manually defined columns of neurons. We defined the boundaries of a column by drawing lines perpendicular to the pial surface of the cortex through the most lateral GFP + neurons in the column. Columns were defined by GFP + cells that had unambiguous pyramidal projection neuron morphologies and were located laterally within $50 \mu \mathrm{m}$ of another pyramidal neuron. We counted the numbers of GFP+ glia inside, near (within $50 \mu \mathrm{m}$ ) and outside of these boundaries (Fig. 1D). Brains that displayed labeled interneurons in addition to labeled projections neurons in cortex were excluded from this analysis.

Nearest neighbor analysis. Sections were imaged with the Neurolucida virtual slice software and imported into Image (NIH). The labeled astrocytes and neurons were manually tagged in ImageJ, which recorded their locations. Custom Matlab software was written that detected the nearest neuronal neighbor to an astrocyte and computed its distance.

Neuronal density as a function of distance. The dataset generated in the nearest neighbor analysis was analyzed using custom Matlab software that measured neuronal density as a function of distance.

We compared the ratio of labeled cells to unlabeled cells within a column and the GFP+ astrocyte to neuron ratio with the overall astrocyte to neuron ratio in cortex using the following techniques. The boundaries of the column were defined as described above, by drawing lines perpendicular to the pial surface of cortex. Astrocytes were identified by S100B expression and neurons via NeuN expression. The somata of cells were counted using a modified version of the stereological optical dissector technique: cells intersecting the upper boundary were excluded from the counts, minimizing overcounting. The top and bottom $3 \mu \mathrm{m}$ of each section were excluded from analysis to reduce sectioning and vibratome chatter artifacts. The variable locations of the columns made assessing absolute numbers of neurons and astrocytes within columns in a stereologically correct manner technically challenging, thus we limited ourselves to assessing ratios of various cell types.

Cluster analysis. Individual columns were imaged as described above and astrocytes were manually tagged using ImageJ. Custom Matlab software determined the number of clusters and the number of astrocytes within a cluster. A cluster was defined as a group of GFP + astrocytes each within $25 \mu \mathrm{m}$ of another GFP+ astrocyte.

\section{Results}

\section{Analysis of cell lineage in the cortex via recombination in transgenic mice}

We generated several lines of enhancer trap mice carrying the enhancer detector cassette thy1.2-cre that expresses Cre recombinase under the transcriptional control of the minimal promoter (129 base pairs) of the mouse Thy-1.2 gene. We crossed these thy1.2-cre mice to the reporters Z/EG (Novak et al., 2000) or mch/lox/GFP mice (gift from S. Dymecki), which, after Cre/ loxP recombination, permanently express GFP. The transgene integrated into different sites in each of the thy1.2-cre lines, giving rise to different patterns of GFP labeling in the brain. One of the thy1.2-cre lines, TFC.09, had a low rate of recombination in a sparse and apparently random subset of neural precursors during development. TFC.09 mice are able to breed, have normal lifespans, and their behavior and brain structure are grossly normal. In the TFC.09 line, a single copy of the provirus integrated into the seventh intron of the glypican- 3 gene. The expression of Cre and the subsequent recombination events in the TFC.09 line showed no correlation with the expression pattern of the glypican-3 gene (unpublished results). While a low recombination efficiency is a common hindrance to the investigators using CRE/LOX systems, it was advantageous in our case, because labeling a small subset of cells in the brain allowed us to examine individual columns and cells, as described below.

We examined $>200$ of the progeny of TFC.09 mice crossed to reporter mice. Approximately $40 \%$ of these mice showed some pattern of GFP labeling in their CNS. In 20\% of the progeny of TFC.09 $\times$ reporter mice, the loxP-recombination gave rise to discrete columnar structures of GFP + cells in the neocortex (Fig. $1 A-C)$. The columns of GFP + cells, which extended radially through layers 1 through 6 , are similar to the columns of clonally related neurons that have been described in the literature (Fig. 1 A-C) (Luskin et al., 1988; Price and Thurlow, 1988; Parnavelas et al., 1991; Mione et al., 1994; Tan et al., 1998; McCarthy et al., 2001; Reid and Walsh, 2002; Wilkie et al., 2004). The GFP+ neurons in these columns had morphologies consistent with projection neurons.

In $\sim 5 \%$ of the progeny of TFC. $09 \times$ lox reporter mice, labeled cells with the morphological characteristics of interneurons were found dispersed broadly throughout the striatum and cortex (Parnavelas et al., 1991; Luskin et al., 1993; Tan et al., 1998; Reid and Walsh, 2002) (Fig. 2A,B). Additionally, we found that some of the progeny of TFC. $09 \times$ reporter mice had GFP labeled neurons in a number of extra-cortical regions, including the hippocampus, thalamus, olfactory bulb, cerebellum, septum and midbrain. These regional patterns of GFP + cells were usually found only in one hemisphere; however, some mice had bilateral labeling, often with different patterns of labeling in each hemisphere. The above patterns of labeling were observed in both the $\mathrm{Z} / \mathrm{EG}$ and $\mathrm{mch} / \mathrm{lox} / \mathrm{GFP}$ reporter mice, indicating that these results are independent of the particular reporter line used. 

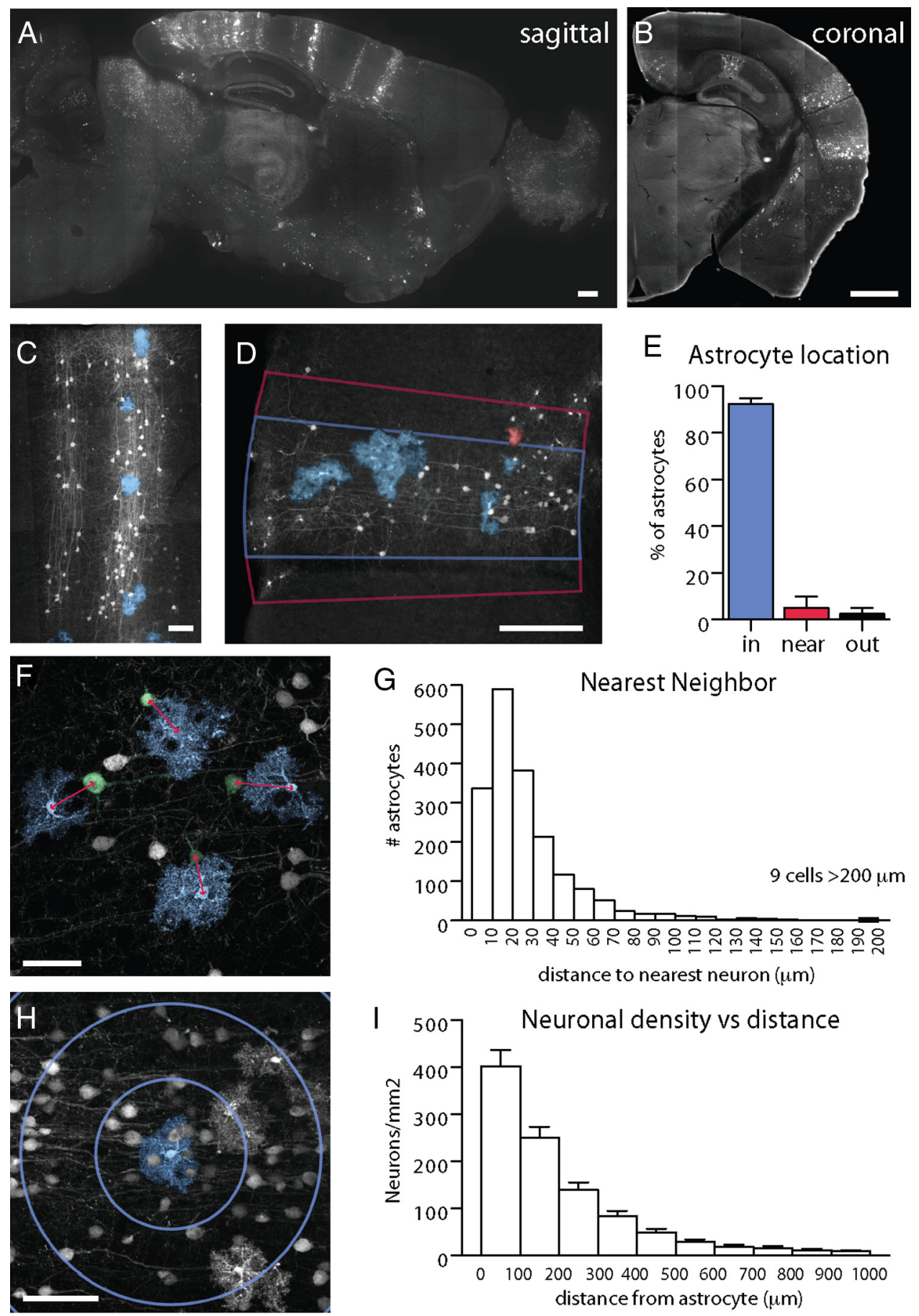

distance to nearest neuron $(\mu \mathrm{m})$
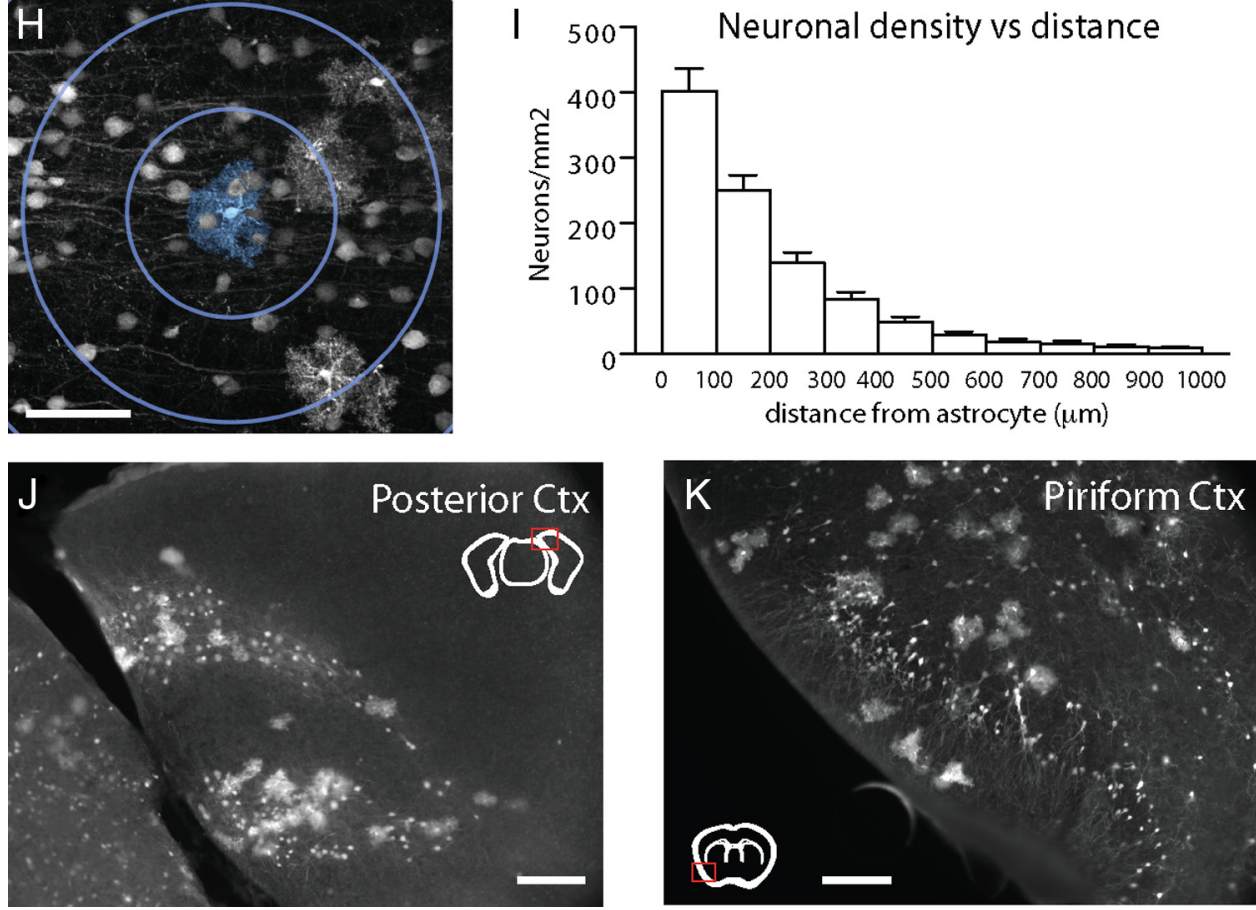

Figure 1. Developmentally generated columns of cells in cortex contain both neurons and astrocytes. $A, B$, Adult TFC. $09 \times$ Z/EG mice exhibit radially arrayed columns of cells in cortex apparent both along the sagittal $(\boldsymbol{A})$ and coronal $(\boldsymbol{B})$ planes. These columns of cells display the characteristic morphologies of developmental columns generated in previous cortical clonal (Figure legend continues.) 

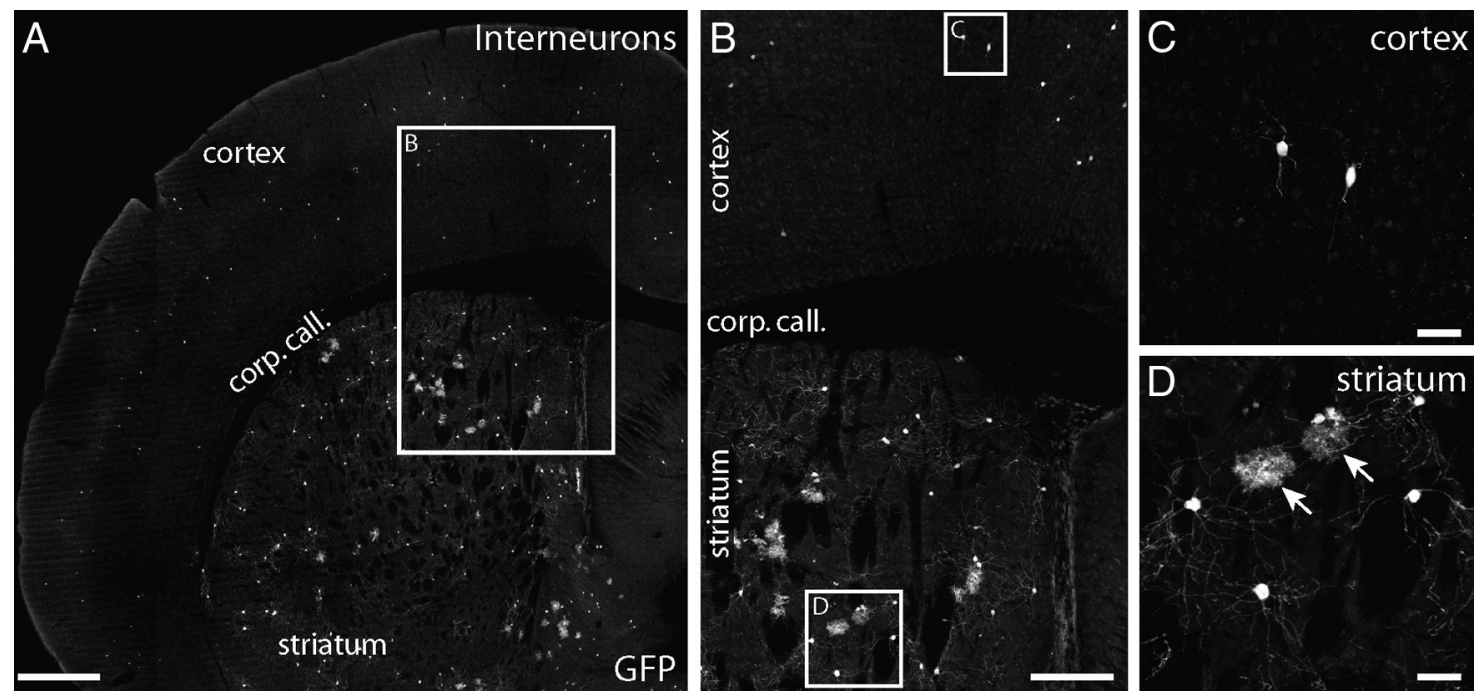

Figure 2. Interneurons in cortex are generated independently of cortical astrocytes. Approximately $5 \%$ of adult TFC.09 $\times$ Z/EG mice exhibited widely distributed GFP + cells within cortex that were not arranged in columns $(\boldsymbol{A})$. In these mice, GFP + cells were distributed sparsely in layers 2 through 6 of neocortex through the entire anteroposterior and mediolateral axes $(\boldsymbol{A}, \boldsymbol{B})$. The GFP + cells in cortex of these mice displayed the characteristic morphologies of interneurons, with fine local processes ( $($ ). The cortices of these mice did not contain labeled astrocytes $(C)$. TFC.09 $\times Z / E G$ mice with interneurons in cortex always had GFP + cells broadly distributed through their ipsilateral striatum $(\boldsymbol{A}, \boldsymbol{B}, \boldsymbol{D})$. In mice with GFP + interneurons in cortex, the striatum contained both GFP + neurons and astrocytes (D). In the striata of these mice, GFP + neurons and astrocytes had the characteristic morphologies of medium spiny interneurons and protoplasmic astrocytes, respectively (D). $C$ and $\boldsymbol{D}$ are high-magnification views of the insets labeled " $C$ " and " $D$ " in $\boldsymbol{B}$. $\boldsymbol{B}$ is a higher-magnification view of the inset in $\boldsymbol{A}$. Scale bars: $\boldsymbol{A}, 500 \mu \mathrm{m} ; \boldsymbol{B}, 250 \mu \mathrm{m} ; \boldsymbol{C}, \boldsymbol{D}, 50 \mu \mathrm{m}$.

\section{Coincident generation of protoplasmic astrocytes and} pyramidal neurons within developmental cortical columns Prior experiments involving retroviral labeling of precursor cells in embryonic animals indicated that few or none of the labeled cortical columns contained both glia and neurons (Luskin et al., 1988; Price and Thurlow, 1988; Parnavelas et al., 1991; Mione et al., 1994; Tan et al., 1998; McCarthy et al., 2001; Reid and Walsh, 2002; Wilkie et al., 2004). However, the columns of labeled cells we observed appeared to contain both neurons and glia (Fig. $1 A-C)$. The GFP + glial cells had typical morphologies of protoplasmic astrocytes and their processes appeared to occupy nonoverlapping domains, as has been previously described (Bushong et al., 2002; Wilhelmsson et al., 2006; Halassa et al., 2007). A total of $97.5 \pm 1.5 \%$. GFP + cells with astrocytic morphologies (of 603 cells examined in $n=3$ mice) expressed S100B (Fig. 3A-C), a protein expressed almost exclusively by astrocytes in cortex. A total of $8.2 \pm 3.7 \%$ of GFP + cells with astrocytic morphologies (611 cells examined in $n=3$ mice) expressed GFAP (Fig. 3D-F),

(Figure legend continued.) labeling experiments using other methods. C, These columns of GFP labeled cells (white) contained both neurons and astrocytes, which have been pseudocolored blue for the purposes of visualization. $\mathbf{C}, \mathbf{D}, \boldsymbol{F}, \mathbf{G}$, We measured whether GFP + astrocytes were in (blue lines), near (within $50 \mu \mathrm{m}$ as indicated by the red lines), or outside of GFP + columns. Columns were defined as discreet, elongated structures in the cortex that contained GFP + cells with unambiguous pyramidal neuron morphologies spanning from the ventricular to the pial surface. $\boldsymbol{F}, \mathbf{G}$, We measured the distance between astrocytes (blue) and their nearest neighboring neuron (green) to assess their spatial relationship. The average distance between an astrocyte and its nearest neighbor was $33 \pm 28 \mu \mathrm{m}$. $\mathbf{H}, \mathbf{I}$, We assessed neuronal density as a function of distance, measuring the density of neurons within $100 \mu \mathrm{m}$ of an astrocyte, 200 $\mu \mathrm{m}, 300 \mu \mathrm{m}$, etc. Astrocytes were found in regions of high neuronal density, as would be expected of astrocytes located within neuronal columns. J, Astrocytes were found associated with neuronal columns even when the columns were curved, as they are commonly in the occipital cortex, suggesting their association is not merely accidental. $\boldsymbol{K}$, In piriform cortex, with its three-layered structure that is distinct from neocortex, neither layer 2 interneurons nor astrocytes exhibited columnar organization, demonstrating that the columnar structures we found in neocortex are unlikely to be artifactual. Scale bars: $A, B, M, N, 500 \mu \mathrm{m} ; \boldsymbol{D}, J, K, 100 \mu \mathrm{m}$; $C, F, H, 50 \mu \mathrm{m}$. a protein expressed in activated astrocytes. GFP + cells with astrocytic morphologies did not express NeuN, a marker of mature neurons (Fig. 3G-I), or MBP, a marker of oligodendrocytes. Thus, both the morphology of these cells and their immunochemical profile indicate that they are astrocytes. GFP + astrocytes exhibited numerous processes that enveloped NeuN+ neurons within the same columnar structures. The neurons encompassed by GFP + astrocytes were often GFP + themselves, suggesting a possible functional relationship between labeled cells (Fig. 3G-I).

To measure the spatial relationship between GFP+ astrocytes and columns of labeled neurons we used three metrics.

First, we defined the boundaries of a column by drawing lines perpendicular to the pial surface of the cortex through the most lateral GFP + neurons in a column and counted the numbers of GFP + glia inside, near (within $50 \mu \mathrm{m}$ ) and outside of these boundaries (Fig. 1D). Columns were defined as discrete, elongated structures in the cortex that contained GFP+ cells with unambiguous pyramidal neuron morphologies spanning from layer 6 to the pial surface. We measured the position of 3509 $\mathrm{GFP}+$ astrocytes relative to $343 \mathrm{GFP}+$ columns in the brains of 9 adult mice and found that $92.6 \pm 3.8 \%$ of astrocytes were found within the boundaries of a column, $6.1 \pm 2.8 \%$ were found within $50 \mu \mathrm{m}$ outside of these boundaries and $1.7 \pm 2.0 \%$ were found further away (Fig. $1 E$ ). These results suggest that GFP+ astrocytes are predominantly located within columns delineated by labeled pyramidal neurons.

Second, to avoid biases due to the manual selection of column boundaries we measured the distance between individual GFP+ astrocytes and the nearest GFP + neuron (Fig. 1 F). We analyzed a total of 1878 astrocytes and 10,944 neurons, marking the position of every GFP + astrocyte and every GFP + neuron in cortex in every 12 th brain section from 5 adult mice. The average distance between a GFP + astrocyte and the nearest GFP + neuron was $33 \pm 28 \mu \mathrm{m}$ (Fig. 1G). A total of $95 \%$ of GFP + astrocytes had their nearest GFP+ pyramidal neuronal neighbor within $80 \mu \mathrm{m}$. 
Third, using the dataset from the second analysis, we constructed histograms to assess the density of GFP + pyramidal neurons within $100,200,300 \mu \mathrm{m}$, etc. of GFP + astrocytes (Fig. $1 \mathrm{H}$ ). The density of labeled pyramidal neurons was highest near labeled astrocytes, and dropped rapidly with distance (Fig. 1I). These data show that GFP + astrocytes are closely associated with regions with high density of GFP+ projection neurons, as in the labeled columns we observed.

Together, these measurements indicate that labeled progenitors form columns of cells in cortex containing both pyramidal neurons and protoplasmic astrocytes. Furthermore, GFP+ astrocytes were found within columns of GFP + neurons even when these columns were not straight, such as the curved column in posterior cortex shown in Figure 1J. The colocalization of astrocytes and neurons in curved columns suggests that their proximity is not merely artifactual or accidental.

\section{Generation of astrocytes outside of the neocortex}

While we detected columns comprised of GFP + pyramidal neurons and astrocytes in the neocortex, we did not find columns of labeled cells in regions of the brain in which columnar patterning has not been described. For example, the piriform cortex has a 3-layered architecture in which layer 2 consists almost exclusively of densely packed interneurons in a pattern reminiscent of granular neurons. In TFC. $09 \times$ lox reporter mice both the labeled layer 2 piriform cortex neurons and the labeled astrocytes were broadly distributed, and showed no columnar organization (Fig. $1 \mathrm{~K}$ ).

We also found mice $(n=11)$ that contained GFP + cells in their neocortex with characteristic interneuron morphologies. In these mice, GFP+ interneurons were found widely distributed throughout both the cortex and the ipsilateral striatum, without any apparent columnar organization (Fig. 2A). These GFP+ neurons were distributed in a pattern similar to that of cells derived from the ganglionic eminences, which generate both striatal and cortical interneurons (Parnavelas et al., 1991; Luskin et al., 1993; Tan et al., 1998; Reid and Walsh, 2002). Whereas all 11 mice had both interneurons and astrocytes labeled in their striata (Fig. $2 B, D$ ), only interneurons were labeled in the cortex (Fig. 2C). GFP+ astrocytes were only present in the striatum when GFP + interneurons were also present in the striatum and cortex. These data suggest that interneurons and striatal astrocytes share a common precursor. Furthermore, these data indicate that the coincident, columnar labeling of GFP + projection neurons and astrocytes in the neocortex of these transgenic mice accurately represents the distribution of the progeny from neuronal and astrocyte progenitors in the brain.

\section{Lineage relationship between neurons and protoplasmic} astrocytes within neocortical columns

To examine whether the GFP + neurons and astrocytes we observed within cortical columns shared a common precursor, we
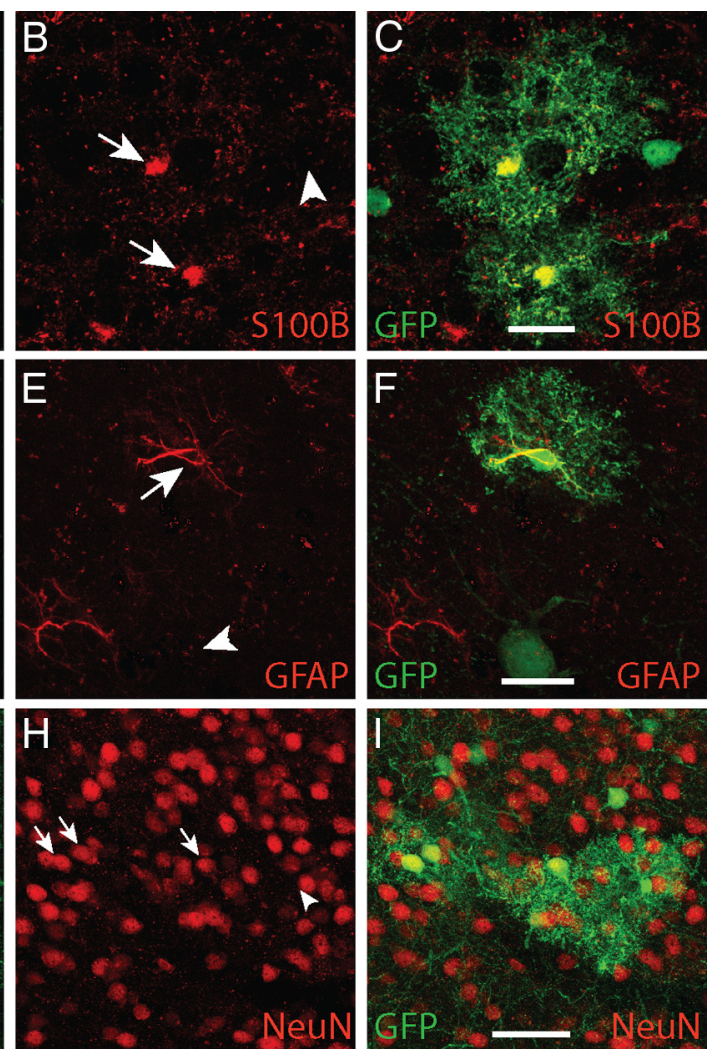

Figure 3. Non-neuronal GFP+ cells displayed characteristics of protoplasmic astrocytes. $\boldsymbol{A}-\boldsymbol{C}$, Non-neuronal GFP + cells in developmental columns in adult mice exhibited characteristic protoplasmic astrocyte morphologies (arrows). D-F, A total of - $1.5 \%$ (603 cells examined in $n=3$ mice) of non-neuronal GFP + cells expressed S100B, an astrocytic marker. A total of ( 611 cells examined in $n=3$ mice) expressed GFAP, a protein expressed in reactive and astrocytes in cortex. G-I, Labeled cells with neuronal morphologies expressed neither S100B nor GFAP (arrowheads). Conversely, GFP + cells with astrocytic morphologies did not express NeuN, a mature neuronal marker. The processes of GFP + astrocytes often encompassed GFP+ neurons. Scale bars: $\boldsymbol{A}-\boldsymbol{F}, 10 \mu \mathrm{m} ; \boldsymbol{G}-\mathbf{I}, 25 \mu \mathrm{m}$.

examined the frequency with which the GFP + pyramidal neurons and GFP + astrocytes overlapped. We found that 95\% (325 of 343 columns, $n=9$ mice) of GFP + columns contained at least one astrocyte. Sixteen columns were found to contain only neurons, and in two instances a columnar structure consisting of only astrocytes was found. The few columns we observed that contained only neurons or only astrocytes had very few GFP + cells, suggesting that, due to the plane of section, we may have been only sampling the edge of the column.

Although it is theoretically possible that GFP + glial columns and GFP+ neuronal columns arose independently from each other in the same locations, this is an unlikely scenario. There are $1 \times 10^{7}$ neurons in the neocortex of the adult mouse (Schüz and Palm, 1989). Approximately $70 \%$ of cortical neurons are projection neurons (Jones, 1993; Wonders and Anderson, 2005). Thus there are $\sim 7 \times 10^{6}$ projection neurons in neocortex. Tan et al., report that cortical columns arise in multiples of 600 , with their largest column containing $\sim 5000$ neurons, and the average column containing $\sim 1800$ neurons (Tan et al., 1998). Dividing $7 \times$ $10^{6}$ by 1800 indicates that there are $\sim 3900$ columns in the cortex of the adult mouse.

According to these estimates, in the 9 mice we examined, there were a total of $\sim 35,000$ columns $(9 \times 3900)$ in their cortices. Of these estimated 35,000 columns, 343 contained GFP + neurons; thus, the probability of finding a column of GFP+ neurons $\left(P_{\mathrm{N}+}\right)$ is 0.01 . A total of 327 of 35,000 columns contained GFP + astrocytes; the probability of finding a column of GFP+ astrocytes 

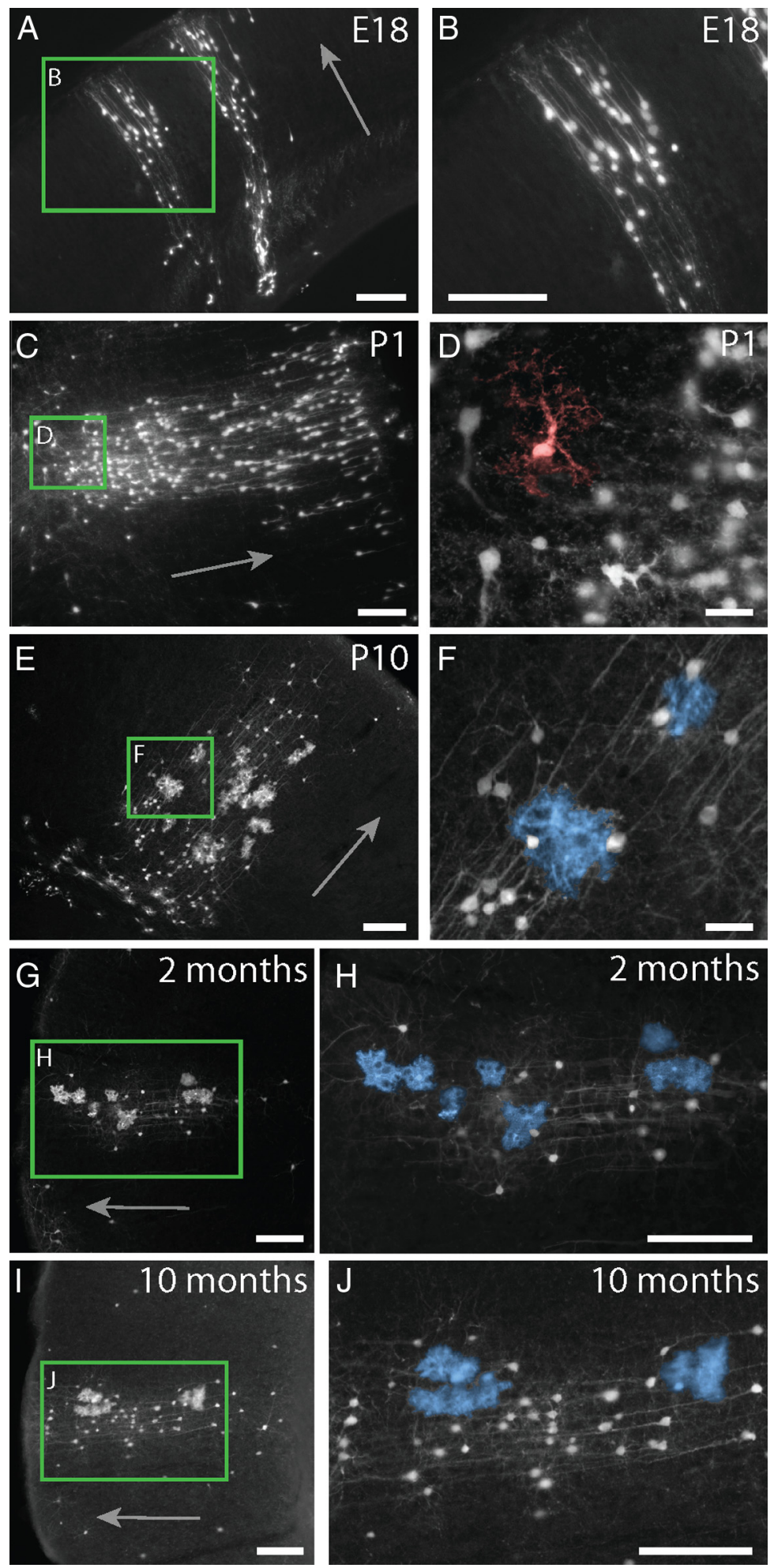

Figure 4. Astrocytes develop within cortical columns and maintain their spatial relationship through adulthood. $\boldsymbol{A}, \boldsymbol{B}$, At E18, columns of radially arrayed projection neurons were visible in cortex. C, D, By P1, GFP + cells with morphologies resembling immature astrocytes (pseudocolored in red) were visible within the columns. $\boldsymbol{E}, \boldsymbol{F}$, At P10, mature astrocytes (pseudocolored in blue in $\boldsymbol{F}$ ) were found within columns distributed through the depth of cortex, much like they are in adult mice. $\boldsymbol{G}, \boldsymbol{H}$, Columns in 2-month-old adult mice contained pyramidal neurons and multiple clusters of multiple astrocytes. GFP + astrocytes were still found closely arrayed with columns of GFP + cortical neurons in 10-month-old mice, indicating that astrocyte addition and/or turnover in the adult mouse does not appear to result in the widespread dispersion of astrocytes. Scale bars: $\boldsymbol{A}-\boldsymbol{C}, \boldsymbol{E}, \boldsymbol{G}-\boldsymbol{J}, 100 \mu \mathrm{m}$; $\boldsymbol{D}, \boldsymbol{F} ; 25 \mu \mathrm{m}$. Mature astrocytes are pseudocolored in blue in $\boldsymbol{E}, \boldsymbol{H}$, and $\boldsymbol{J}$. An immature astrocyte is pseudocolored in red in $\boldsymbol{D}$. Arrows in $\boldsymbol{A}, \boldsymbol{C}, \boldsymbol{E}, \boldsymbol{G}$, and $\boldsymbol{I}$ point toward the pial surface of the neocortex.
$\left(P_{\mathrm{A}+}\right)$ is 0.01 . Thus, the probability that independent events would generate a column containing both GFP + neurons and astrocytes is $P_{\mathrm{N}+} \times P_{\mathrm{A}+}$, or 0.0001 . The likelihood that a given GFP + column of neurons would contain independently generated GFP + astrocytes is $\left(P_{\mathrm{N}+} \times\right.$ $\left.P_{\mathrm{A}+}\right) / P_{\mathrm{N}+}, 0.01$ or $1 \%$. The most parsimonious explanation for the $95 \%$ overlap we saw between GFP+ neurons and GFP+ astrocytes is that the neurons and astrocytes in these columns are generated from a shared precursor cell.

We examined the ratio of labeled cells to unlabeled cells within a column using the optical fractionator method. GFP+ astrocytes comprised $10.5 \pm 1.1 \%$ of all $\mathrm{S} 100 \mathrm{~B}+$ astrocytes within a column (10 columns from each of $n=3$ mice). GFP + neurons accounted for $11.7 \pm 3.3 \%$ of all $\mathrm{NeuN}+$ neurons within a column ( $10 \mathrm{col}-$ umns from each of $n=3$ mice). Thus, the precursor that generates a developmental column makes a similar contribution to both populations of cells; it generates $\sim 10 \%$ of both neurons and astrocytes within a given cortical column.

We compared the GFP+ astrocyte to $\mathrm{GFP}+$ neuron ratio with the overall astrocyte to neuron ratio in cortex. We found the ratio of GFP + astrocytes to GFP + pyramidal neurons was 1:7.4 \pm 2.9 (50 randomly selected columns sampled from $n=5$ mice). The overall ratio of $\mathrm{S} 100 \mathrm{~B}+$ astrocytes to NeuN+ neurons was 1:8.4 \pm 1.0 (50 randomly selected columns sampled from $n=5$ mice). NeuN + cells include both pyramidal neurons and interneurons in cortex. Our astrocyte to neuron ratios are similar to the 1:6 astrocyte to neuron ratio in motor cortex and the 1:8 ratio in sensory cortex previously reported (Irintchev et al., 2005). Since pyramidal projection neurons generated in columns account for the $\sim 70 \%$ of the neurons in cortex, these results suggest that most cortical astrocytes are derived from developmental columns as well.

\section{Dynamics of astrocyte generation in neocortical columns}

Prior in vivo experiments suggested that radial glia transform directly into individual astrocytes as neurogenesis comes to an end (Schmechel and Rakic, 1979; Voigt, 1989; Culican et al., 1990; Gressens et al., 1992). Such data would neatly explain the colocalization of a single astrocyte with each developmentally generated column of neurons in the neocortex. However, most labeled developmental cortical columns contained multiple clusters, each comprised of multiple astrocytes (Figs. 

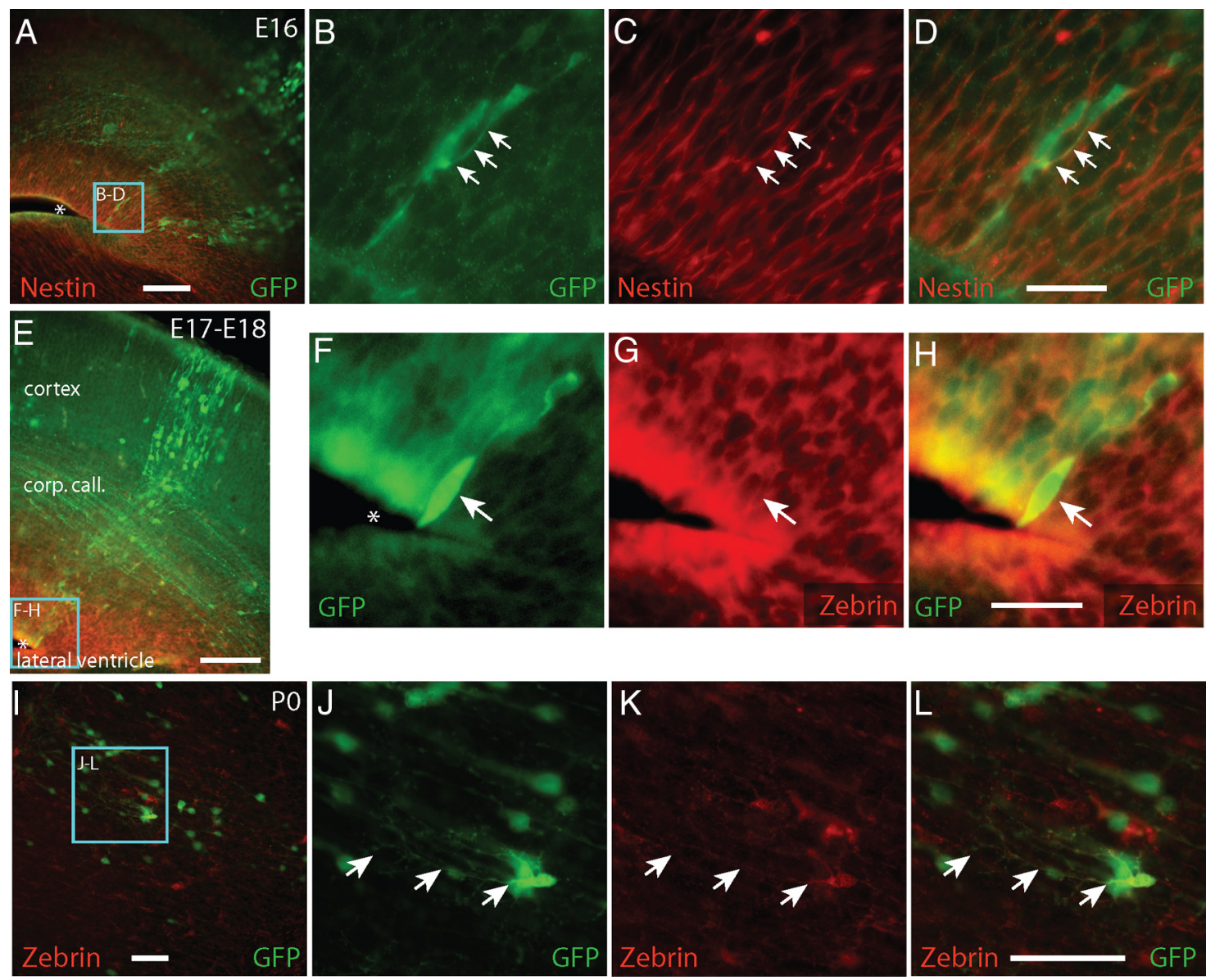

Figure 5. GFP + cells express radial glia markers zebrin and nestin during development. Between E16 and E18, GFP + cells form columns of extending from the ventricles to the pia. GFP + cells (green) near the ventricles in these columns expressed Nestin $(\boldsymbol{A}-\boldsymbol{D}$, red) or Zebrin $(\boldsymbol{E}-\boldsymbol{H}$, red), proteins expressed by radial glia. By P0, zebrin $+/ \mathrm{GFP}+$ cells were found in the parenchyma of cortex, away from the ventricular wall, presenting morphologies resembling a shortened radial glia. $\boldsymbol{A}-\boldsymbol{D}$, Arrows indicate GFP +/Nestin + cells. $E-L$, Arrows indicate GFP +/zebrin + processes and cells. $A, E, F$, Asterisks indicate the neocortical ventricular wall. Zebrin +/GFP + cells were absent by P4, a time by which most radial glia have disappeared from the brain. Scale bars, $50 \mu \mathrm{m}$.

$1 C, D, J, 3 A, G, 4 E, G, J)$. There were an average of $2.7 \pm 1.5$ clusters of GFP + astrocytes per column (114 columns examined from $n=5$ mice). A cluster was defined as a group of GFP+ astrocytes each within $25 \mu \mathrm{m}$ of another GFP+ astrocyte. Each cluster contained between 1 and 15 astrocytes, with an average of $3.6 \pm 2.9$ astrocytes per cluster (139 clusters counted from 50 columns in $n=5$ mice). These counts are likely underestimates, because they are based on counting cells in a single $50 \mu \mathrm{m}$ thick section through each column, and columns generally extended through multiple sections. Nonetheless, the distribution of labeled astrocytes we observe is unlikely to be explained by the transformation of single radial glia into an individual astrocyte.

We followed the development of cells present in the GFP+ columns in the cortex by examining mice at embryonic day 18 (E18), postnatal day 0 (P0), P1, P2, P4, P6, P8, P10, young adult mice (2 months old), and older adult mice (10 months old). At $\mathrm{E} 18$ and P1, GFP + columns in cortex contained exclusively pyramidal neurons, and we did not detect any GFP + cells with mature astrocytic morphologies in cortex (Fig. $4 A-D$ ). This observation is consistent with previous works indicating that cortical gliogenesis occurs predominantly in the postnatal period. In contrast, subcortical gliogenesis was apparent by $\mathrm{P} 1$, a time at which we could already detect labeled cells displaying the morphologies of mature protoplasmic astrocytes in the striatum. At $\mathrm{P} 1$, we detected columns in the neocortex that contained, in addition to GFP + pyramidal neurons, GFP + cells that resembled the immature astrocytes previously described in cortex (Fig. 4D) (Zerlin and Goldman, 1997). By P4, we found that columns contained both GFP + pyramidal neurons and GFP + cells with mature astrocytic morphologies. Thus, the astrocytes observed in these transgenic mice developed along the well established timeframe of astrocyte generation. Columns in P10 mice appeared to contain more astrocytes than in younger mice (Fig. $4 E, F$ ) and closely resembled the columns we observed in 2-month-old adult mice, which included GFP + pyramidal neurons and multiple clusters of GFP+ astrocytes (Fig. 4G,H). GFP+ astrocytes in more mature adults (10 months old) were still found almost exclusively within developmental cortical columns consisting of both GFP+ pyramidal neurons and astrocytes (Fig. 4I,J). Thus, astrocyte addition and/or turnover in the adult brain does not lead to their dispersion outside of the developmental columns in which they originate, as they maintained their spatial relationships well into adulthood.

To examine the relationship between radial glia and the GFP+ developmental columns, we examined the expression of nestin and zebrin, two proteins commonly used to identify radial glia. At E16 and E17, toward the end of cortical neurogenesis, we found columns of cells extending from the ventricle (Fig. 5A), through the corpus callosum, to the pial surface of cortex (Figs. $4 A, 5 E$ ). This organization reflects the well characterized organization of cortical columns previously described (Tan et al., 1998). During the late embryonic period (E16-E18), a subset of GFP + cells near 
the bases of these columns expressed nestin and zebrin. The example included in Figure 5, $A-D$, shows a GFP + cell with nestinpositive proximal processes at E16. The example GFP+/zebrin + cell in Figure 5, E-H, was found lining the ventricle at E17 and exhibited zebrin-positive perinuclear staining. By P0, GFP +/zebrin + cells were found in the parenchyma of cortex away from the ventricular wall (Fig. 5J-L). Previous studies have described cells similar to these ones we observed, with morphologies resembling shortened radial glia (Marshall and Goldman, 2002). It has been suggested that these cells may be in the process of differentiating from radial glia into protoplasmic astrocytes (Marshall and Goldman, 2002). These results suggest that some, if not all, the GFP + cells located in the columns we observe are descendants of GFP+ radial glia.

To identify the location of dividing cells during the period of gliogenesis, we treated mice with BrdU, a thymidine analog that integrates into the DNA of dividing cells, at P0, P2, P4, P6, or P8 and killed them $4 \mathrm{~h}$ later. At P0 $(n=5$ mice), we found 13 $\mathrm{GFP}+/ \mathrm{BrdU}+$ cells at the bases of columns and $2 \mathrm{GFP}+/ \mathrm{BrdU}+$ cells within the cortical layers (Fig. $6 A-D)$. At $\mathrm{P} 2(n=3$ mice) and $\mathrm{P} 4(n=5$ mice $)$ we found 21 and $8 \mathrm{GFP}+/ \mathrm{BrdU}+$ cells within the cortical layers, respectively. At P2 and P4, we did not find GFP $+/ \mathrm{BrdU}+$ cells at the bases of columns. The example illustrated in Figure 6, $E-G$, contains $\mathrm{GFP}+/ \mathrm{BrdU}+$ cells in both layers 2 and 6 of P2 cortex. By P6 ( $n=3$ mice), GFP $+/ \mathrm{BrdU}+$ cells in developmental columns were infrequent or absent. Dividing GFP + cells were found near the ventricle early in development and within the cortical layers later.

In the proliferation experiments, we found two P0 mice and one P4 mouse that exclusively had GFP+ cells with interneuron morphologies distributed widely throughout cortex. While mice with GFP + columns had both GFP+ pyramidal neurons and astrocytes in their cortices, mice with GFP + interneurons did not exhibit GFP+ astrocytes in their cortices. Mice with selective labeling of interneurons had no GFP $+/ \mathrm{BrdU}+$ cells in their cortices, suggesting that the postnatal GFP $+/ \mathrm{BrdU}+$ cells are in the astrocyte lineage.

To analyze the fate of GFP + dividing cells during the postnatal period, we treated $\mathrm{P} 0$ mice with $\mathrm{BrdU}$ and killed them at $\mathrm{P} 4$ (Fig. $6 \mathrm{H}-\mathrm{K}$ ). We found GFP $+/ \mathrm{BrdU}+$ cells that had adopted astrocytic morphologies throughout the depth of cortex by $\mathrm{P} 4$ $(n=5)$. These GFP $+/ \mathrm{BrdU}+$ cells were often found in pairs, suggesting that they underwent their most recent division near their final location.

In summary, dividing cells were found near the bases of columns during early postnatal gliogenesis and within the cortical layers thereafter, suggesting a pattern of cell proliferation and radial migration that could account for the regionally specific distribution of astrocytes in the mature cortex.

\section{Discussion}

We examined the development of cell lineages in neocortex using a transgenic mouse that undergoes apparently random recombination in a small subset of cells during embryogenesis, yielding GFP + cells that can be followed from embryonic development through adulthood. We found developmental columns of GFP+ cells in neocortex consisting of both projection neurons and protoplasmic astrocytes. Ninety-five percent of labeled columns, as defined by the distribution of GFP + pyramidal neurons in a radially oriented structure spanning from the ventricular to the pial surface, also contained GFP + protoplasmic astrocytes. In addition, almost all of the labeled astrocytes in the neocortex were found in or near developmental columns of labeled neurons. The ratio of GFP + astrocytes to GFP + neurons in columns was $\sim 1$ : 7.4, which is similar to the overall ratio of astrocytes to neurons in cortex, 1:8.4, and suggests that most cortical astrocytes originate in developmental columns. After birth, some GFP + cells incorporated BrdU during the period of gliogenesis in a pattern suggestive of a precursor that underwent an initial division in the germinal zone and subsequent divisions within more superficial cortical layers. Most of the resulting columns contained multiple clusters of several astrocytes. Our results indicate that a significant population of cortical astrocytes is generated in a spatially restricted manner, a process that may contribute to the diversity and regional specification of astrocytes.

We observed columns of labeled cells in the neocortex that are similar to those previously described (Luskin et al., 1988; Tan and Breen, 1993; Mione et al., 1994; Tan et al., 1998; McCarthy et al., 2001; Reid and Walsh, 2002; Wilkie et al., 2004). However, in contrast to previous experiments, we found that our columns consisted of both astrocytes and neurons. We examined the spatial relationship between astrocytes and neurons and found that $98 \%$ of astrocytes were in or within $50 \mu \mathrm{m}$ of a column (as defined by the presence of radially oriented clusters of GFP + pyramidal neurons) and, on average, GFP + astrocytes were within 33 $\mu \mathrm{m}$ of their nearest GFP+ neuron. Previous research may have been unable to detect astrocytes within developmental columns due to technical limitations and/or the comparatively small number of mice and columns examined. For instance, the promoters used in previous efforts may have driven robust gene expression only in neurons. In addition, older labeling techniques, such as lacZ labeling, do not fill the cytoplasm entirely, making identification of cell types more challenging.

Several pieces of evidence indicate that the colocalization of labeled neurons and astrocytes in developmental columns accurately represents the physiological pattern of development of neurons and astrocytes in the cortex. First, GFP+ neurons and astrocytes were found together in developmental columns even when the columns are curved, reducing the likelihood that the labeled cells are colocalized merely due to chance. Second, regions of the brain in which columnar patterning has not been described do not show colocalization of GFP+ neurons and astrocytes in discrete patterns. For instance, piriform cortex contained both widely distributed GFP+ layer 2 interneurons and GFP + astrocytes, without any obvious pattern. Third, a subset of mice exhibited GFP+ interneurons but no developmental columns of GFP+ projection neurons in cortex. In these mice, GFP + interneurons were always found broadly distributed both in the striatum and throughout neocortex, reproducing previous findings about neurons derived from the ganglionic eminences (Parnavelas et al., 1991; Luskin et al., 1993; Tan et al., 1998; Reid and Walsh, 2002). However, even though these mice exhibited $\mathrm{GFP}+$ astrocytes in their striata and GFP+ interneurons in their cortices, their cortices did not contain any GFP + astrocytes. These results show that TFC.09 mice recapitulate well established developmental patterns of brain development and indicate that astrocytes in cortex are generated by spatially restricted progenitors, whose progeny display little tangential dispersion.

Radial glia simultaneously function both as a scaffold supporting the migration of projection neurons, and as progenitor cells generating new neurons in the neocortex (Noctor et al., 2001; Tamamaki et al., 2001; Malatesta et al., 2003; Anthony et al., 2004). Each radial glia generates neurons that migrate along their radial process into cortex, forming a developmental column of projection neurons, and previous work indicates that clonally related pyramidal neurons in the cortex are arranged in develop- 

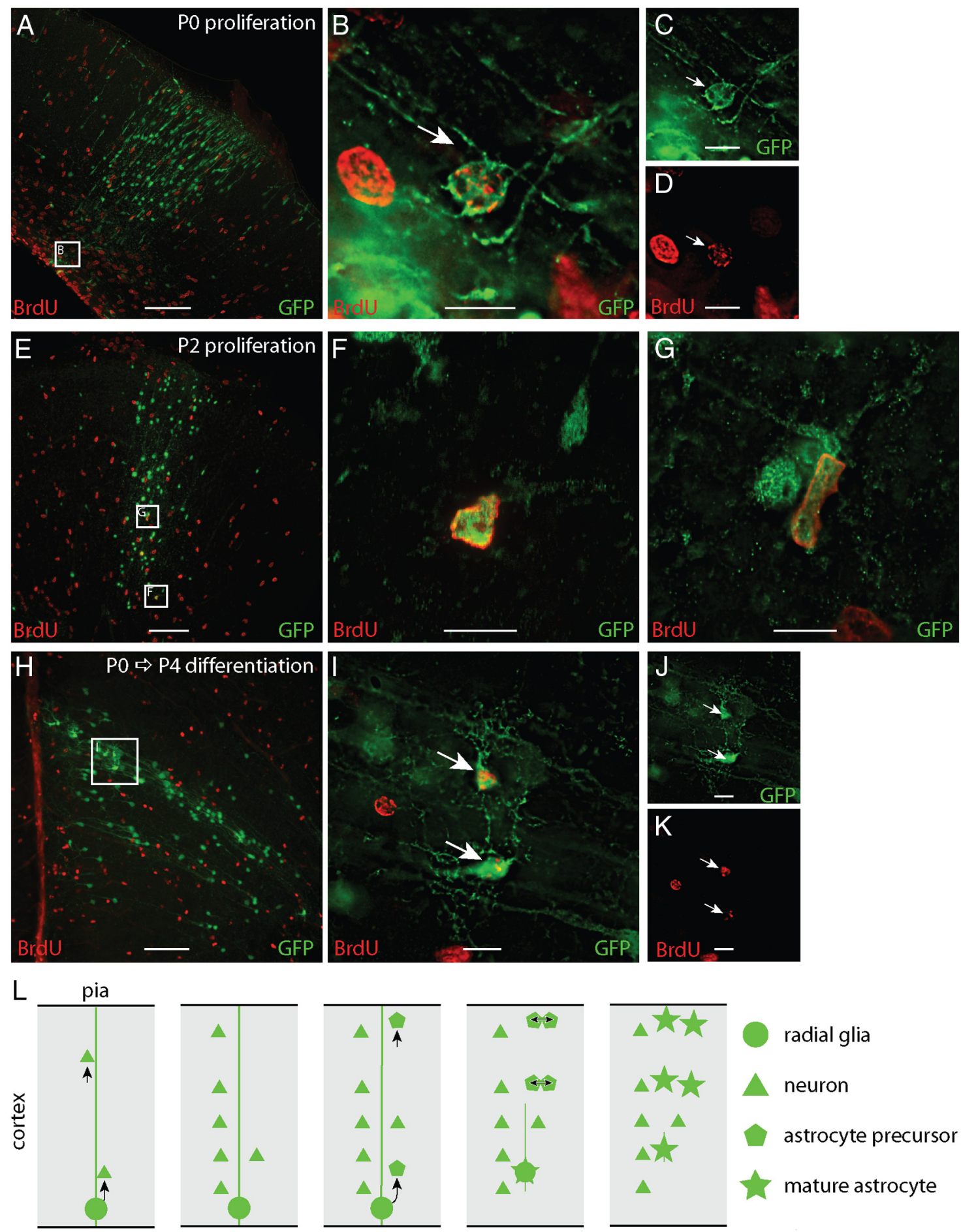

radial glia

ventricle

time $\rightarrow$

Figure 6. Astrocyte precursors proliferate within cortical columns. To identify proliferating cells, mice were treated with BrdU and killed 4h later. A-D, At P0, dividing, BrdU + (red), GFP + (green) cells were most frequently found at the bases of cortical columns, nearest the ventricle. $E-G$, By P2, the majority of dividing GFP + cells were distributed within cortex and few were found near the bases of columns. To examine the fate of dividing GFP + cells, we treated mice with BrdU at P0 and killed them at P4.GFP + cells labeled with BrdU at PO were found through the depth of cortex. $\boldsymbol{H}-\boldsymbol{K}, \mathrm{GFP}+/ \mathrm{BRDU}+$ cells were also often found in pairs, suggesting that they differentiated near the site of their final division. These results suggest the following model of cortical astrogenesis $(\boldsymbol{L})$ : Radial glia first generate columns of projection neurons in cortex. Subsequently, the radial glia generate several astrocytic precursors that distribute radially along the column of neurons. These precursors undergo their final divisions in the different layers of the cortex, forming multiple clusters of multiple astrocytes within a developmental cortical column. Scale bars: $A, E, H, 100 \mu \mathrm{m} ; \boldsymbol{B}-\boldsymbol{D}, \boldsymbol{F}, \mathbf{G}, \mathbf{I}-\boldsymbol{K}, 10 \mu \mathrm{m}$.

mental columns (Luskin et al., 1988; Parnavelas et al., 1991; Mione et al., 1994; Tan et al., 1998; McCarthy et al., 2001). We found that $95 \%$ of GFP + columns, as defined by the distribution of radial clusters of GFP + pyramidal neurons, also contained
GFP + astrocytes. If labeled neuronal columns and astrocytic columns arose completely independently, from separate progenitors, their spatial overlap would be expected to be much lower, $\sim 1 \%$, as discussed above. Our data indicate that the appearance 
of labeled neuronal and astrocytic columns are not independent events. The observed overlap is best explained by a progenitor that gives rise to both neurons and astrocytes within a developmental column.

The origin of cortical astrocytes is not well understood. It has been postulated that both radial glia and the subventricular zone (SVZ) may give rise to astrocytes in the neocortex. Prior in vivo experiments suggested that radial glia retract their processes and differentiate into protoplasmic astrocytes after neurogenesis came to an end. Individual radial glia were thought to transform into individual astrocytes (Schmechel and Rakic, 1979; Voigt, 1989; Culican et al., 1990). In contrast, in vitro experiments suggested that radial glia can proliferate after neurogenesis and generate multiple astrocytes (Malatesta et al., 2000). In contrast to both these findings, experiments tracing the generation of developmental columns of cortical projection neurons found no associated astrocytes (Luskin et al., 1988; Price and Thurlow, 1988; Parnavelas et al., 1991; Mione et al., 1994; Tan et al., 1998; McCarthy et al., 2001; Reid and Walsh, 2002; Wilkie et al., 2004). The apparent inconsistencies in these data concerning the radial glial contribution to astrocyte development were difficult to reconcile.

In addition to the reported small number of astrocytes derived from radial glia, several works suggested that the majority of astrocytes were derived from SVZ precursors that dispersed widely to populate cortex. As neurogenesis comes to an end, cells from the SVZ were thought to proliferate, generating oligodendrocyte and astrocyte precursors (Levison et al., 1993; Levison and Goldman, 1993; Luskin and McDermott, 1994; Marshall and Goldman, 2002) that migrated from the SVZ and dispersed widely through cortex (Zerlin et al., 1995; Kakita and Goldman, 1999; Kakita et al., 2003). These experiments were performed by injecting retroviruses into the SVZ of early postnatal mice and assessing the locations and phenotypes of labeled cells. Injecting viruses into the brain induces injury, which could perturb the migration patterns and fate of cells labeled. Gliosis is a common response to injury and could potentially increase the number of astrocytes labeled in the cortex after a retroviral injection into the SVZ. In addition, the timing of these injections, after neurogenesis has been completed, likely explains why these experiments labeled glial cells, but no neurons.

Our data indicate that precursors originating in developmental columns generate the majority of cortical astrocytes. Within a developmental column, there is 1 GFP+ astrocyte for every 7.4 GFP + pyramidal neurons. In the cortical gray matter overall, there is 1 astrocyte for every 8.4 neurons. Since pyramidal neurons, which in mice are exclusively generated in developmental columns, comprise $\sim 70 \%$ of the neurons in cortex (Wonders and Anderson, 2005), these data suggest that protoplasmic astrocytes associated with columns comprise the majority of protoplasmic astrocytes in cortex.

Our experiments indicate a pattern of neocortical gliogenesis in which proliferation of astrocyte precursors occurs initially at the base of columns and later occurs within the cortical layers. At $\mathrm{P} 0$, dividing GFP + cells are found at the base of columns near the ventricle. At P2 and P4, dividing GFP + cells are found within the cortical layers. At P2, some columns contained multiple dividing GFP + cells in different layers of cortex. Precursors labeled with BrdU at P0 frequently formed closely located pairs of astrocytes within cortex by $\mathrm{P} 4$, supporting the hypothesis they underwent their final division in cortex. Consistent with these findings, columns in adult mice frequently contained multiple clusters each consisting of multiple astrocytes.
Together our data suggest the following model (Fig. $6 \mathrm{~L}$ ): radial glia give rise to both projection neurons and protoplasmic astrocytes in the mammalian neocortex. After generating a column of neurons, the radial glia generates multiple astrocyte precursors. These astrocyte precursors distribute through the depth of cortex, migrating radially within the neuronal column. They undergo their final divisions in the layers of the cortex, differentiate into protoplasmic astrocytes and form clusters of cells that respect the boundaries of the neuronal column.

Our findings suggest a developmental process that could confer positional identity upon astrocytes along the axes of the neocortex. Cortical astrocytes serve essential roles in the development of synapses and in modifying neurotransmission (Pfrieger and Barres, 1997; Allen and Barres, 2005; Volterra and Meldolesi, 2005; Zhang and Haydon, 2005; Stevens, 2008). Thus it has been hypothesized that cortical astrocytes are likely to be regionally specified like cortical neurons (Reisin and Colombo, 2002; Wilhelmsson et al., 2006; Hochstim et al., 2008; Allen and Barres, 2009). Consistent with this hypothesis, it has been shown that spinal cord astrocytes are regionally specified in the spinal cord (Muroyama et al., 2005). Establishing whether astrocytes in the neocortex exhibit region-specific differences and examining the potential relationship between these astrocytes and their neighboring neurons within columns will be of particular interest in the future.

\section{References}

Allen NJ, Barres BA (2005) Signaling between glia and neurons: focus on synaptic plasticity. Curr Opin Neurobiol 15:542-548.

Allen NJ, Barres BA (2009) Neuroscience: Glia-more than just brain glue. Nature 457:675-677.

Anthony TE, Klein C, Fishell G, Heintz N (2004) Radial glia serve as neuronal progenitors in all regions of the central nervous system. Neuron 41:881-890.

Bushong EA, Martone ME, Jones YZ, Ellisman MH (2002) Protoplasmic astrocytes in CA1 stratum radiatum occupy separate anatomical domains. J Neurosci 22:183-192.

Costa MR, Bucholz O, Schroeder T, Götz M (2009) Late origin of gliarestricted progenitors in the developing mouse cerebral cortex. Cereb Cortex 19 [Suppl 1]:i135-i143.

Culican SM, Baumrind NL, Yamamoto M, Pearlman AL (1990) Cortical radial glia: identification in tissue culture and evidence for their transformation to astrocytes. J Neurosci 10:684-692.

Gressens P, Richelme C, Kadhim HJ, Gadisseux JF, Evrard P (1992) The germinative zone produces the most cortical astrocytes after neuronal migration in the developing mammalian brain. Biol Neonate 61:4-24.

Halassa MM, Fellin T, Takano H, Dong JH, Haydon PG (2007) Synaptic islands defined by the territory of a single astrocyte. J Neurosci 27:6473-6477.

Hochstim C, Deneen B, Lukaszewicz A, Zhou Q, Anderson DJ (2008) Identification of positionally distinct astrocyte subtypes whose identities are specified by a homeodomain code. Cell 133:510-522.

Irintchev A, Rollenhagen A, Troncoso E, Kiss JZ, Schachner M (2005) Structural and functional aberrations in the cerebral cortex of tenascin-C deficient mice. Cereb Cortex 15:950-962.

Jones EG (1993) GABAergic neurons and their role in cortical plasticity in primates. Cereb Cortex 3:361-372.

Kakita A, Goldman JE (1999) Patterns and dynamics of SVZ cell migration in the postnatal forebrain: monitoring living progenitors in slice preparations. Neuron 23:461-472.

Kakita A, Zerlin M, Takahashi H, Goldman JE (2003) Some glial progenitors in the neonatal subventricular zone migrate through the corpus callosum to the contralateral cerebral hemisphere. J Comp Neurol 458:381-388.

Levison SW, Goldman JE (1993) Both oligodendrocytes and astrocytes develop from progenitors in the subventricular zone of postnatal rat forebrain. Neuron 10:201-212.

Levison SW, Chuang C, Abramson BJ, Goldman JE (1993) The migrational 
patterns and developmental fates of glial precursors in the rat subventricular zone are temporally regulated. Development 119:611-622.

Luskin MB, McDermott K (1994) Divergent lineages for oligodendrocytes and astrocytes originating in the neonatal forebrain subventricular zone. Glia 11:211-226.

Luskin MB, Pearlman AL, Sanes JR (1988) Cell lineage in the cerebral cortex of the mouse studied in vivo and in vitro with a recombinant retrovirus. Neuron 1:635-647.

Luskin MB, Parnavelas JG, Barfield JA (1993) Neurons, astrocytes, and oligodendrocytes of the rat cerebral cortex originate from separate progenitor cells: an ultrastructural analysis of clonally related cells. J Neurosci 13:1730-1750.

Malatesta P, Hartfuss E, Götz M (2000) Isolation of radial glial cells by fluorescent-activated cell sorting reveals a neuronal lineage. Development 127:5253-5263.

Malatesta P, Hack MA, Hartfuss E, Kettenmann H, Klinkert W, Kirchhoff F, Götz M (2003) Neuronal or glial progeny: regional differences in radial glia fate. Neuron 37:751-764.

Marshall CA, Goldman JE (2002) Subpallial dlx2-expressing cells give rise to astrocytes and oligodendrocytes in the cerebral cortex and white matter. J Neurosci 22:9821-9830.

McCarthy M, Turnbull DH, Walsh CA, Fishell G (2001) Telencephalic neural progenitors appear to be restricted to regional and glial fates before the onset of neurogenesis. J Neurosci 21:6772-6781.

Mione MC, Danevic C, Boardman P, Harris B, Parnavelas JG (1994) Lineage analysis reveals neurotransmitter (GABA or glutamate) but not calcium-binding protein homogeneity in clonally related cortical neurons. J Neurosci 14:107-123.

Muroyama Y, Fujiwara Y, Orkin SH, Rowitch DH (2005) Specification of astrocytes by bHLH protein SCL in a restricted region of the neural tube. Nature 438:360-363.

Noctor SC, Flint AC, Weismman TA, Dammerman RS, Kriegstein AR (2001) Neurons derived from radial glial cells establish radial units in neocortex. Nature 409:714-720.

Novak A, Guo C, Yang W, Nagy A, Lobe CG (2000) Z/EG, a double reporter mouse line that expresses enhanced green fluorescent protein upon Cremediated excision. Genesis 28:147-155.

Parnavelas JG, Barfield JA, Franke E, Luskin MB (1991) Separate progenitor cells give rise to pyramidal and nonpyramidal neurons in the rat telencephalon. Cereb Cortex 1:463-468.

Pfrieger FW, Barres BA (1997) Synaptic efficacy enhanced by glial cells in vitro. Science 277:1684-1687.

Price J, Thurlow L (1988) Cell lineage in the rat cerebral cortex: a study using retroviral-mediated gene transfer. Development 104:473-482.
Reid CB, Walsh CA (2002) Evidence of common progenitors and patterns of dispersion in rat striatum and cerebral cortex. J Neurosci 22:40024014.

Reisin HD, Colombo JA (2002) Considerations on the astroglial architecture and the columnar organization of the cerebral cortex. Cell Mol Neurobiol 22:633-644.

Schmechel DE, Rakic P (1979) A Golgi study of radial glial cells in developing monkey telencephalon: morphogenesis and transformation into astrocytes. Anat Embryol (Berl) 156:115-152.

Schüz A, Palm G (1989) Density of neurons and synapses in the cerebral cortex of the mouse. J Comp Neurol 286:442-455.

Stevens B (2008) Neuron-astrocyte signaling in the development and plasticity of neural circuits. Neurosignals 16:278-288.

Tamamaki N, Nakamura K, Okamoto K, Kaneko T (2001) Radial glia is a progenitor of neocortical neurons in the developing cerebral cortex. Neurosci Res 41:51-60.

Tan SS, Breen S (1993) Radial mosaicism and tangential cell dispersion both contribute to mouse neocortical development. Nature 362:638-640.

Tan SS, Kalloniatis M, Sturm K, Tam PP, Reese BE, Faulkner-Jones B (1998) Separate progenitors for radial and tangential cell dispersion during development of the cerebral neocortex. Neuron 21:295-304.

Voigt T (1989) Development of glial cells in the cerebral wall of ferrets: direct tracing of their transformation from radial glia into astrocytes. J Comp Neurol 289:74-88.

Volterra A, Meldolesi J (2005) Astrocytes, from brain glue to communication elements: the revolution continues. Nat Rev Neurosci 6:626-640.

Wilhelmsson U, Bushong EA, Price DL, Smarr BL, Phung V, Terada M, Ellisman MH, Pekny M (2006) Redefining the concept of reactive astrocytes as cells that remain within their unique domains upon reaction to injury. Proc Natl Acad Sci U S A 103:17513-17518.

Wilkie AL, Jordan SA, Sharpe JA, Price DJ, Jackson IJ (2004) Widespread tangential dispersion and extensive cell death during early neurogenesis in the mouse neocortex. Dev Biol 267:109-118.

Wonders C, Anderson SA (2005) Cortical interneurons and their origins. Neuroscientist 11:199-205.

Zerlin M, Goldman JE (1997) Interactions between glial progenitors and blood vessels during early postnatal corticogenesis: blood vessel contact represents an early stage of astrocyte differentiation. J Comp Neurol 387:537-546.

Zerlin M, Levison SW, Goldman JE (1995) Early patterns of migration, morphogenesis, and intermediate filament expression of subventricular zone cells in the postnatal rat forebrain. J Neurosci 15:7238-7249.

Zhang Q, Haydon PG (2005) Roles for gliotransmission in the nervous system. J Neural Transm 112:121-125. 